\title{
Cholesterol-Granuloma in the Middle Ear
}

\begin{tabular}{|l|l|l|}
\hline A.J.K. & Korthals & Altes \\
\hline
\end{tabular}

Utrecht

Author's address: A. J. Korthals Altes M. D., E. N. T. Clinic, University of Utrecht, Utrecht (Netherlands)

In two cases of exploration of the middle ear in children, indication for the operation was a hearing loss of $40 \mathrm{db}$ of the conduction type, a little tumor in the medial side of the eardrum was found.

The histological picture is composed of granulation tissue, cholesterin cristals, the blood pigment hemosiderin, fibrin and giant cells of the foreign bodytype.

This cases of cholesterol-granuloma are sporadically described in the middle ear. In the mastoid cells these granulomata are more common in combination with brown to black liquid and a blue eardrum.

The three possibilities of genesis:

crystallization in glue, rich of cholesterol, crystallization in an old bleeding,

fat degeneration of leucocytes.

Although rare in otology, elsewhere in the body these cholesterol-crystals are rather common.

We find these crystals formed by fat degeneration in atheromatous cysts, dermoid cysts and periapical cysts of the jaws and in the xanthomatous degeneration in antibiotic masked mastoiditis.

In our patients we found indications of a bleeding.

Conclusion

In two cases of catarrhal otitis media a bleeding occurred that afterwards organized itself to cholesterol-granulomata. 